\title{
Clinical effectiveness of combined interventional therapy as a salvage modality for unresectable pancreatic carcinoma
}

\author{
SUSHANT KUMAR DAS $^{1 *}$, JIN LIANG WANG $^{2 *}$, BING LI $^{1}$, CHUAN ZHANG $^{1}$ and HAN FENG YANG ${ }^{1}$ \\ ${ }^{1}$ Department of Interventional Radiology, Affiliated Hospital of North Sichuan Medical College, Nanchong, Sichuan 637000; \\ ${ }^{2}$ Department of Radiology, Pingdingshan Fifth People's Hospital, Pingdingshan, Henan 467000, P.R. China
}

Received September 20, 2018; Accepted March 21, 2019

DOI: $10.3892 / \mathrm{ol} .2019 .10323$

\begin{abstract}
Although different treatment methods have been introduced to treat advanced pancreatic carcinoma, the median overall survival rate remains unsatisfactory. Theoretically, combining different treatment methods should work in synergy to enhance locoregional disease control and improve survival. Therefore, the aim of the present retrospective study was to analyze the effectiveness of combined interventional therapy compared with trans-arterial chemoembolization (TACE) or chemotherapy alone for the treatment of unresectable pancreatic carcinoma. A total of 266 patients who were undergoing treatment for unresectable pancreatic carcinoma between July 2012 and November 2015 were included in the current study. The tumor responses and 3-year overall survival rates of patients treated with combined interventional therapy (TACE combined with iodine-125 seed implantation and/or radiofrequency ablation; CIT group; $n=84$ ) were compared with those of patients treated with TACE alone (TACE group; $\mathrm{n}=59$ ), as well as patients treated with systemic chemotherapy alone (control group; $n=123$ ). Patients in the CIT group exhibited significantly improved tumor responses compared with patients in the TACE group (51.89 vs. $30.61 \%$; $\mathrm{P}=0.028)$ or control group (51.89 vs. 17.20\%; $\mathrm{P}<0.001)$. The 3 -year overall survival rate of the CIT group was also significantly higher compared with that of the TACE and control groups ( $\mathrm{P}=0.0116$ and $\mathrm{P}=0.0001$, respectively). Furthermore, the CIT group exhibited a significantly higher overall survival rate for patients with unresectable metastatic pancreatic cancer compared with the TACE and control groups $(\mathrm{P}=0.0088$ and $\mathrm{P}<0.0001$, respectively), which suggests that a combination of
\end{abstract}

Correspondence to: Professor Han Feng Yang, Department of Interventional Radiology, Affiliated Hospital of North Sichuan Medical College, 63 Wenhua Road, Nanchong, Sichuan 637000, P.R. China

E-mail: yhfctjr@yahoo.com

*Contributed equally

Key words: pancreatic carcinoma, trans-arterial chemoembolization, radiofrequency ablation, iodine-125 radioactive seeds different interventional techniques increases the survival of patients with unresectable pancreatic cancer. No life-threatening complications were observed in any treatment group. In conclusion, combined interventional therapy exhibits a good efficacy and an improved survival rate for unresectable pancreatic cancer compared with TACE alone.

\section{Introduction}

According to the American Cancer Society estimates in 2014, pancreatic carcinoma is the fourth most common cause of cancer-associated mortality in the USA (1). Pancreatic carcinoma is characterized by insidious onset, rapid progression, a high degree of malignancy, early metastasis and poor prognosis (1). The disease has a poor prognosis due to a lack of early diagnostic symptoms. As a result, the majority of patients are diagnosed with mid- and terminal-staged pancreatic cancer, which cannot be surgically removed (2). Although $15-20 \%$ of patients are diagnosed at an early stage, only $20-25 \%$ of these patients may survive $>5$ years after tumor resection (3). Despite the introduction of new therapeutic techniques, including external beam radiotherapy, intraoperative radiotherapy, radioactive seed implantation and chemotherapy, the prognosis for patients with pancreatic carcinoma remains unsatisfactory with a 5-year survival rate of $<6 \%$ (4).

Autopsy studies have suggested that $8-15 \%$ of patients succumb to the disease due to the local destructive power of pancreatic carcinoma rather than systemic metastatic spread, according to different pattern of genetic mutations (5-7). Thus, there is a limited response to chemotherapy in patients with pancreatic carcinoma. This has led to the development and application of an ablative technique in pancreatic cancer termed radiofrequency ablation (RFA) (7). The most common worldwide application of RFA for pancreatic cancer is for the treatment of patients with stage III disease, either as an initial strategy at the time of diagnosis or when there is no response to standard systemic treatments (8-10). However, RFA can also be used in patients with stage IV metastatic disease to induce positive modulation of the immune system (11-13). Recently, the application of RFA as an initial treatment method has been proposed on the basis of a presumed immunological antitumor stimulation induced by RFA; a randomized control trial is currently being performed to evaluate this (14). In a systemic review by Rombouts et al (15), the RFA-associated morbidity 
rate has been reported to range between 4.0 and $17.8 \%$, and the RFA-associated mortality rate ranges between 0.0 and $3.0 \%$ when a maximal ablation temperature of $90^{\circ} \mathrm{C}$ is used. Furthermore, the median survival time reported following RFA was 25.6 months (15).

In addition, different new intervention techniques, including transarterial chemotherapy, computed tomography (CT)-guided iodine-125 ( $\left.{ }^{125} \mathrm{I}\right)$ seed implantation and radio-immunotherapy, have been investigated (16-19). CT-guided percutaneous implantation of ${ }^{125} \mathrm{I}$ seeds provides positional precision with minimal invasion and maintains the slow and continuous release of ${ }^{125} \mathrm{I}$ seeds, which has been suggested to be radio-biologically advantageous, allowing the repair of non-lethal damage and re-oxygenation of hypoxic areas in normal tissues $(20,21)$. A number of studies have confirmed the safety of this technique with a mortality rate of $0.006-0.031 \%$ and a complication rate of $0.5-3.0 \%(22,23)$. Furthermore, in a study by Yu et al (18), the incidence rates of complications were not statistically different between the normal visceral organs group and the visceral organs punctured group. Additionally, patients with 3-4 punctures and patients with 5-4 punctures did not exhibit a significant difference in adverse events, which suggests that ${ }^{125}$ I seed implantation may be a comparatively safer technique.

Trans-arterial chemoembolization (TACE) is another interventional technique that has been has been demonstrated to be efficient for the control of both symptoms and tumor masses in patients with pancreatic cancer (24). TACE is a locoregional procedure that provides a highly concentrated dose of chemotherapeutic drug to tumor cells, prolonging drug-cell contact time and minimizing systemic toxicity (25). Despite the emergence of these aforementioned interventional techniques, to the best of our knowledge, there is currently no consensus regarding the best therapeutic modality for unresectable pancreatic carcinoma. Therefore, it is necessary to investigate novel techniques that may improve patient outcome. The present study analyzed the effectiveness of combined interventional therapy (CIT; TACE combined with ${ }^{125}$ I seed implantation and/or RFA) compared with TACE alone for the treatment of unresectable pancreatic carcinoma.

\section{Materials and methods}

Patients. Patients with unresectable pancreatic carcinoma, who were treated either with TACE alone or with TACE combined with CT-guided percutaneous implantation of ${ }^{125} \mathrm{I}$ seeds and/or radiofrequency ablation at the Interventional Radiology Department of the Affiliated Hospital of North Sichuan Medical College (Nanchong, China) and Pingdingshan Fifth People's Hospital (Pingdingshan, China) between July 2012 and November 2015, were included in the present retrospective study. Patients who did not receive any interventional therapy and were treated with systemic chemotherapy were considered as the control group. The criteria for enrollment were as follows: i) Ductal adenocarcinomas of the pancreas that were pathologically diagnosed by CT-guided fine needle aspiration prior to interventional procedures; ii) contraindication to curative resection because of locally advanced primary tumor or the presence of liver metastasis; iii) presence of measurable lesions in the liver or pancreas that corresponded to Response Evaluation Criteria in Solid Tumors (26) target lesions; iv) Karnofsky physical score (KPS) $\geq 60$; and v) age $\geq 18$ years $(27)$. The exclusion criteria were: i) patients with extra hepatic metastasis; ii) coagulation dysfunction and platelet count $<50 \times 10^{9}$ per liter; iii) local infection or uncontrollable systemic infection; and iv) contraindication for interventional therapy due to severe liver, kidney or cardiac dysfunction. A total of 266 patients with an average age (mean \pm standard deviation) of $67.24 \pm 12.46$ years met the inclusion criteria and were enrolled in the present study. Of these patients, 162 were male and 104 female. The current study was approved by the Institutional Review Board of Affiliated Hospital of North Sichuan Medical College (Nanchong, China) as well as Pingdingshan Fifth People's Hospital (Pingdingshan, China). Written informed consent was obtained from all the patients.

Procedure for TACE. Gemcitabine-based trans-arterial chemotherapy is a type of therapeutic strategy recognized by the Chinese Society of Clinical Oncology-Pancreatic Cancer Professional Committee $(28,29)$. Trans-arterial chemotherapy is a routine treatment for patients with advanced pancreatic cancer in China (28).

In the present study, the Seldinger technique (30) was used under local anesthesia to access the femoral artery with a 5-Fr vascular sheath. Subsequently, a digital subtraction angiography examination [Infinix-i core ${ }^{+}$(INFX-800V) Canon Medical Systems, Tustin, CA, USA] was performed following catheterization of the celiac and superior mesenteric arteries with a 5.0-Fr (Radiofocus ${ }^{\circledR}$ Angiographic catheter; Terumo, Tokyo, Japan) or (Torcon ${ }^{\circledR}$ NB Advantage; Catheter Cook Medical, Bloomington, IN, USA) catheter. Segmental or sub-segmental tumor-feeding artery was selected using either 2.0-Fr (Progreat ${ }^{\circledR}$; Terumo) or a 3.0-Fr (Renegade ${ }^{\mathrm{TM}}$; Boston Scientific Corporation, Marlborough, MA, USA) micro-catheter depending upon the diameter of the selected vessel. Emulsions of iodized oil (Lipiodol Ultra-Fluid; André Guerbet Laboratories, Aulnay Sous-Bois, France), 100 mg/m² Gemcitabine and $100 \mathrm{mg} / \mathrm{m}^{2}$ Oxaliplatin (both from Jiangsu Hengrui Medicine Co., Ltd, Lianyungang, China) were infused. The amount of emulsion was decided based on tumor size and vascularity.

The body and tail of the pancreas are supplied by the dorsal pancreatic artery, the great pancreatic artery and the caudal pancreatic artery; all of which generally originate from the splenic artery $(31,32)$. Therefore, in the current study, in case of a lesion in the pancreatic body or tail, a full dose of chemotherapeutic drug was infused via the splenic artery if the aforementioned arteries originated from the splenic artery. In patients with lesions in the head of the pancreas, one-third of the drug was infused via the super-mesenteric artery and the other two-thirds were infused via the gastroduodenal artery. In cases where the super-mesenteric artery contributed to the tumor blood supply, one-third of the drug was administered via the superior mesenteric artery and two-thirds via the splenic artery. Chemotherapeutic drug was infused via the celiac artery if the tumor blood-supplying arteries originated from the common hepatic artery or the celiac artery. Furthermore, if the tumor blood-supplying arteries were directly super-selected, 
the drugs were infused via the blood-supplying arteries using 3-Fr catheters.

For liver metastases, TACE was performed during the same session as pancreatic primary tumor TACE. Depending on the tumor arterial supply, the tip of the catheter was advanced into the right or left hepatic artery to perform selective arterial embolization.

Procedure for ${ }^{125}$ I seed implantation. CT scans (Siemens AG, Munich, Germany) were performed to calculate the total volume of each tumor using a treatment planning system, which was then used to calculate the expected seed number to be implanted. Under CT guidance, the number and angle of the needle directions (18-gauge; length, 150-200 mm; Dr Japan Co., Ltd, Tokyo, Japan) were calculated according to the puncture approach and tumor size. Subsequently, ${ }^{125}$ I seeds (Model-6711; Beijing Atom and High Technique Industries Inc., Beijing, China), with a half-life of 59.4 days, a low energy level of $27.4 \mathrm{KeV}$ and a half-value layer of $0.025 \mathrm{~mm}$ of lead, were implanted within the tumor maintaining a spacing of $1.0 \mathrm{~cm}$ intervals. Fig. 1 presents representative images from a patient undergoing ${ }^{125} \mathrm{I}$ seed implantation combined with TACE for unresectable pancreatic carcinoma with liver metastases.

Procedure for RFA. Percutaneous RFA was performed with the patient under conscious sedation under CT guidance. Histological confirmation of the diagnosis was obtained in all the patients prior to RFA. RFA was performed with a RF generator (Elektrotom HF $106^{\circledR}$; Berchtold, Tuttlingen, Germany), generating 40-60 W of power. Prior to needle insertion, the point of entry was planned to ensure a safe trajectory and end position. CT scan image guidance was used to precisely place the ablation probes percutaneously within the tumor. The tumor size, location and geometry were considered when selecting whether a single 17-gauge or triple cluster 17-gauge needle electrode would be applied in the RFA procedure. In total, $\sim 4,200 \mathrm{~W}$ of energy was delivered using a saline perfused needle with the aim of ablating the entire tumor along with a $1.0 \mathrm{~cm}$ ablative margin.

Systemic chemotherapy. Gemcitabine $\left(1,000 \mathrm{mg} / \mathrm{m}^{2}\right)$ diluted in normal saline was intravenously administered for $30 \mathrm{~min} /$ week for the first seven weeks (i.e. on day 1, 8, 15, 22, 29, 36 and 43) followed by one-week rest. In subsequent cycles, all patients were administered treatment on days 1, 8 and 15 of every four weeks (4-week cycle) until disease progression, unacceptable toxic effects, or withdrawal of consent. Dose reductions to 750,550 and $425 \mathrm{mg} / \mathrm{m}^{2}$ were allowed for management of adverse events.

Assessment of tumor response and pain relief. The treatment response was evaluated 6 months after the interventional procedure using the modified Response Evaluation Criteria in Solid Tumors (26). A complete response (CR) was defined as disappearance of any intra-humoral arterial enhancement in all lesions; a partial response (PR) was defined as a $\geq 30 \%$ decrease in the sum of the diameters of viable (contrast enhancement in the arterial phase) lesions; progressive disease (PD) was defined as $\mathrm{a} \geq 20 \%$ increase in the sum of diameters of viable lesions; and stable disease (SD) was defined as any cases that did not qualify as either PR or PD (26). The response rate was the sum of CR and PR.

Pain intensity was evaluated and graded by the Numerical Rating Scale (NRS) (33). NRS score of 1-3 was defined as mild pain, 4-6 was defined as moderate pain and 7-10 was defined as severe pain. A good response was defined as severe or moderate pain decreasing to no pain post-treatment (NRS score 6-10 reducing to NRS score 0 ). A medium response was regarded as severe pain reducing to mild pain (NRS score 7-10 reducing to NRS score 1-3) with pain-free sleep. A mild response was regarded as severe pain reducing to moderate (NRS score 7-10 reducing to NRS score 4-6) or a moderate pain reducing to mild (NRS score 4-6 reducing to NRS score 1-3) following treatment. A poor response indicated that there was no change in the severity of pain compared with pre-treatment status (34).

Follow-up and survival rate. Patients were followed up at 3 month intervals during the first year and at 6 month intervals thereafter until the end of the study (36 months) or until death. Follow-up tests included routine hematological, biochemical and serological tests, as well as abdominal CT scans. Survival was calculated from the date of diagnosis to the date of mortality or last follow-up. Local recurrence was defined as tumor progression within the implanted area or surrounding regions according to CT images. Local recurrence and distant metastases were scored until patient mortality and censored thereafter.

Toxicity and complications. Safety was defined according to the frequency of procedural and procedure-associated post-procedural complications. These were evaluated based on common terminology criteria for adverse events (version 4.0) (35).

Statistical analysis. All statistical analyses were performed using Statistical Package for the Social Science (SPSS) version 23.0 (IBM Corp., Armonk, NY, USA) and GraphPad Prism version 5.0 (GraphPad Software, Inc., La Jolla, CA, USA). Continuous variables are presented as the mean \pm standard deviation and categorical variables are presented as percentages. For comparisons of clinical characteristics of the patients between the three groups, one-way analysis of variance (ANOVA) was used to compare continuous variables and a $\chi^{2}$ test was used to compare categorical variables. A $\chi^{2}$ test was also used to compare treatment response outcomes between the groups. Overall survival curves were produced using the Kaplan-Meier method and compared using the log-rank test. $\mathrm{P}<0.05$ was considered to indicate a statistically significant difference.

\section{Results}

Clinical characteristics of the patients. The clinical characteristics of all patients involved in the present study are presented in Table I. According to the Union for International Cancer Control 2002 staging criteria (36), 84 cases were at stage III and 182 cases presented with stage IV pancreatic cancer. A total of 143 patients exhibited a tumor in the pancreatic head and 123 presented with a tumor in the pancreatic body or tail. The average diameter of the tumors was $99.2 \pm 1.23 \mathrm{~mm}$. 

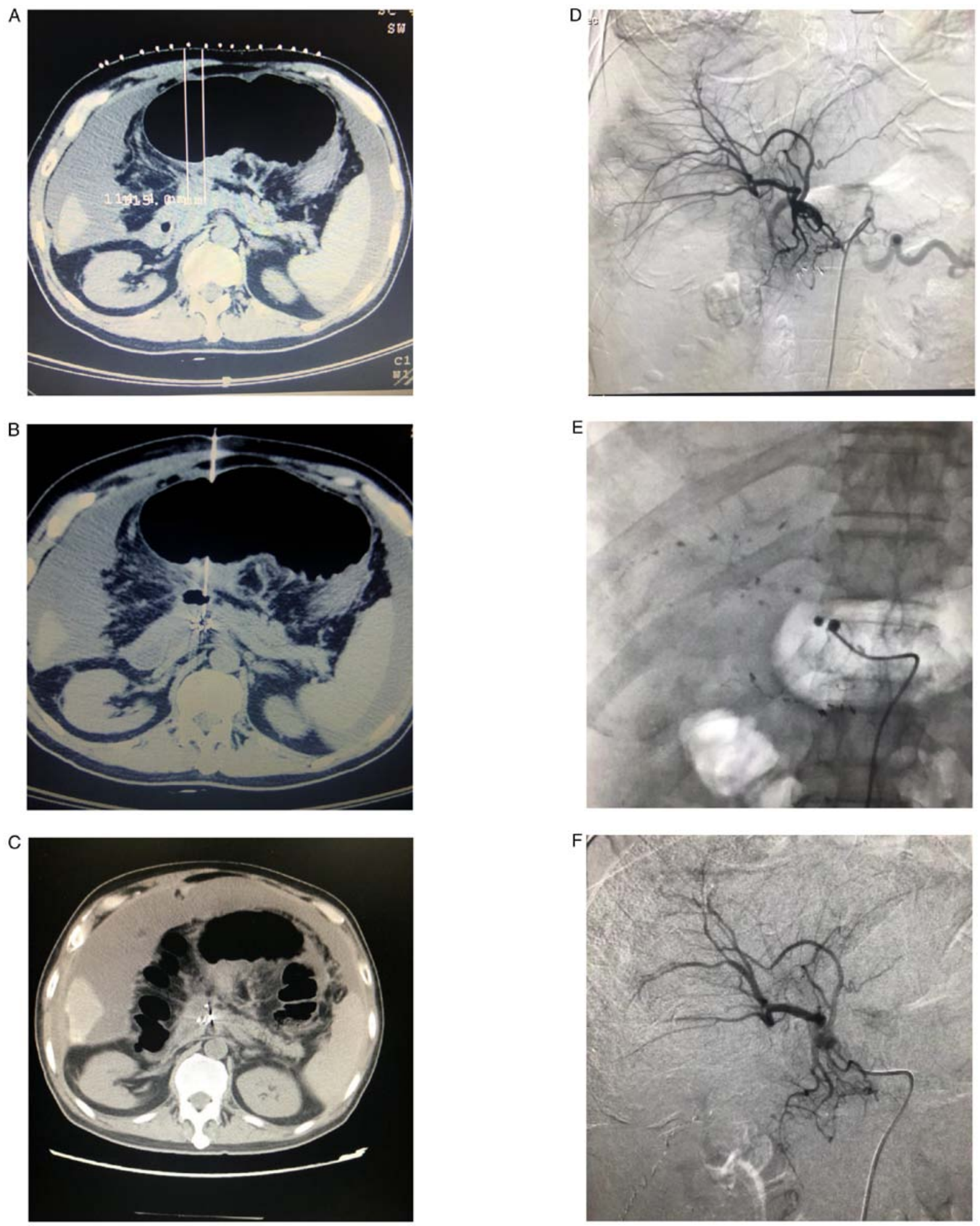

Figure 1. Representative images from a 55 year-old male patient undergoing ${ }^{125}$ I seed implantation combined with TACE for unresectable pancreatic head carcinoma with multinodular liver metastases. (A) Based on the treatment planning system, a needle puncture (anterior approach) with two needles $\sim 1 \mathrm{~cm}$ apart was made. (B) An 18-gauge needle was inserted and ${ }^{125}$ I seeds were then loaded and released every 5-10 mm apart whilst withdrawing the needles. (C) A follow-up computed tomography scan performed 2 months post-procedure revealed a significant decrease in tumor size. (D) The patient was also treated with TACE for primary tumor as well as liver metastases. Angiography through common hepatic artery demonstrating staining of primary as well as metastatic tumor. (E) Visible controlled delivery of emulsion of iodized oil and chemotherapeutic agent to the tumor. (F) An absence of tumor enhancement post-TACE was observed. ${ }^{125}$ I, iodine-125; TACE, trans-arterial chemoembolization.

The main clinical manifestations were epigastric discomfort, abdominal pain and jaundice. The KPS values of 21 patients were between 60 and 69,84 patients had values of $70-79$ and 161 patients presented with values $\geq 80$.

\section{Treatment protocol}

Primary tumor. Of the 266 patients, 143 underwent interventional therapy whereas 123 underwent systemic therapy. The patients who underwent systemic therapy were labeled as the 
Table I. Clinical characteristics of the patients.

\begin{tabular}{|c|c|c|c|c|}
\hline Variable & Control $(n=123), n(\%)$ & TACE $(n=59), n(\%)$ & $\operatorname{CIT}(\mathrm{n}=84), \mathrm{n}(\%)$ & P-value \\
\hline $\begin{array}{l}\text { Age, years } \\
\text { (mean } \pm \text { standard deviation) }\end{array}$ & $66.41 \pm 12.53$ & $68.13 \pm 13.08$ & $66.74 \pm 15.14$ & 0.17 \\
\hline Sex & & & & 0.07 \\
\hline Male & $71(57.72)$ & $38(64.40)$ & $53(63.10)$ & \\
\hline Female & $52(42.28)$ & $21(35.60)$ & $31(36.90)$ & \\
\hline Tumor location & & & & 0.65 \\
\hline Head & $69(56.10)$ & $27(45.76)$ & $47(55.90)$ & \\
\hline Body/tail & $54(43.90)$ & $22(44.24)$ & $37(54.10)$ & \\
\hline UICC stage ${ }^{a}$ & & & & 0.45 \\
\hline Stage III & $55(44.71)$ & $11(18.64)$ & $18(21.42)$ & \\
\hline Stage IV & $68(55.29)$ & $48(81.36)$ & $66(78.58)$ & \\
\hline Size, $\mathrm{cm}$ & & & & 0.24 \\
\hline$<5.0$ & $37(30.00)$ & $10(16.94)$ & $17(20.23)$ & \\
\hline$\geq 5.0$ & $86(70.00)$ & $49(83.06)$ & 67 (79.77) & \\
\hline Ascites & & & & 0.10 \\
\hline Yes & $19(15.44)$ & $4(6.78)$ & $5(6.00)$ & \\
\hline No & $104(84.56)$ & $55(93.22)$ & $79(94.00)$ & \\
\hline Jaundice & & & & 0.14 \\
\hline Yes & $30(24.39)$ & $22(37.28)$ & $29(34.52)$ & \\
\hline No & $93(75.61)$ & $37(62.72)$ & $55(65.48)$ & \\
\hline Child-Pugh classification ${ }^{\mathrm{b}}$ & & & & 0.12 \\
\hline A & $0(0.00)$ & $0(0.00)$ & $0(0.00)$ & \\
\hline B & $34(27.64)$ & $17(28.81)$ & $33(39.28)$ & \\
\hline $\mathrm{C}$ & $89(72.36)$ & $42(71.19)$ & $51(60.72)$ & \\
\hline Karnofsky physical scores ${ }^{c}$ & & & & 0.08 \\
\hline $60-69$ & $12(9.75)$ & $4(6.78)$ & $5(5.95)$ & \\
\hline $70-79$ & $22(17.89)$ & $28(47.46)$ & $34(40.48)$ & \\
\hline$\geq 80$ & $89(72.36)$ & $27(45.76)$ & $45(53.57)$ & \\
\hline Elevated CA 19-9 level & $93(75.60)$ & $48(81.35)$ & $64(76.19)$ & 0.43 \\
\hline
\end{tabular}

${ }^{a}$ Morin E, Cheng S, Mete O, et al: Hormone profiling, WHO 2010 grading, and AJCC/UICC staging in pancreatic neuroendocrine tumor

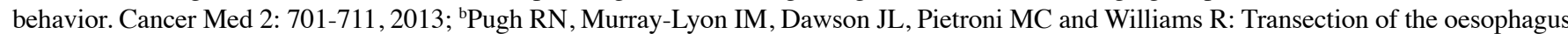
for bleeding oesophageal varices. Br J Surg 60: 646-649, 1973; ${ }^{\circ}$ Karnofsky DA and Burchenal JH: Evaluation of Chemotherapeutic Agents. The clinical evaluation of chemotherapeutic agents in cancer. In: Macleod CM, editor. Columbia University Press 196, 1949. TACE, trans-arterial chemoembolization; CIT, combined interventional therapy; UICC, Union for International Cancer Control; CA 19-9, carbohydrate antigen 19-9.

control group in the present study. Patients undergoing interventional therapy were further divided into the TACE group $(n=59)$, who underwent TACE alone, and the CIT group $(n=84)$, who underwent TACE + RFA and/or ${ }^{125}$ I seed implantation. In the CIT group, 28 patients had undergone TACE + RFA and 47 had undergone TACE $+{ }^{125} \mathrm{I}$. The remaining 9 patients were treated with TACE + RFA $+{ }^{125} \mathrm{I}$.

All patients who underwent TACE had a mean of three TACE sessions per patient (range, 2-6), in four-week or eight-week intervals. The mean ablation session per patient was two (range, 1-3). ${ }^{125}$ I seed implantation was performed only once per tumor.

Metastases. There was a total of 68 unresectable metastatic (UR-M) and 55 unresectable locally advance tumor(UR-L) patients in the control group. In the TACE group, there were 48 UR-M and 11 UR-L patients, whereas, in the CIT group, 66 were UR-M and 18 UR-L. Out of the 68 UR-M patients in the control group, 30 had oligo nodular liver metastases ( $\leq 3$ liver lesions), while 9 had multi nodular liver metastases (>3 liver lesions). No local regional therapy was performed for the liver metastatic tumor in the control group other than systemic chemotherapy. Out of the 48 UR-M patients in the TACE group, 31 had oligo nodular liver metastases, while 17 had multinodular liver metastases. All UR-M patients in the TACE group received TACE for liver metastasis. In the CIT group, of the 66 UR-M patients, 29 had oligo nodular and 37 had multinodular liver metastasis. Of the 66 UR-M patients in the CIT group, 49 patients underwent TACE for liver metastases. A total of 9 patients received ${ }^{125} \mathrm{I}$ seed 
Table II. Tumor response to treatment.

Variable

Control (n=93a $)$ TACE (n=49a) CIT (n=79a) P-value (CIT vs. control) P-value (CIT vs. TACE)

Treatment response, $\mathrm{n}(\%)$

$\begin{array}{lccccc}\text { CR } & 7(7.53) & 9(18.37) & 22(27.84) & <0.001^{\mathrm{b}} & 0.291 \\ \text { PR } & 9(9.67) & 6(12.24) & 19(24.05) & 0.014^{\mathrm{b}} & 0.168 \\ \text { PD } & 34(36.55) & 12(24.48) & 16(20.25) & 0.019^{\mathrm{b}} & 0.661 \\ \text { SD } & 43(46.23) & 22(44.89) & 23(29.11) & 0.020^{\mathrm{b}} & 0.086 \\ \text { ORR }(\%) & 17.20 & 30.61 & 51.89 & <0.001^{\mathrm{b}} & 0.028^{\mathrm{a}}\end{array}$

${ }^{a}$ Total number of patients alive at six aemonth from the initiation of treatment were included for tumor response to treatment evaluation. ${ }^{b} \mathrm{P}<0.05$. TACE, trans-arterial chemoembolization; CIT, combined interventional therapy; CR, complete response; PR, partial response; PD, progressive disease; SD, stable disease; ORR, overall response rate; OS, overall survival; MST, mean survival time.

Table III. Survival analysis.

\begin{tabular}{|c|c|c|c|c|c|}
\hline Variable & Control $(n=123)$ & TACE $(n=59)$ & $\operatorname{CIT}(n=84)$ & P-value (CIT vs. control) & P-value (CIT vs. TACE) \\
\hline \multicolumn{6}{|c|}{ OS rate, months $(\%)$} \\
\hline 12 & 23.57 & 47.46 & 67.85 & $<0.001^{\mathrm{a}}$ & $0.011^{\mathrm{a}}$ \\
\hline 24 & 9.57 & 25.42 & 39.28 & $<0.001^{\mathrm{a}}$ & $0.013^{\mathrm{a}}$ \\
\hline 36 & 4.06 & 11.86 & 22.62 & $<0.001^{\mathrm{a}}$ & $0.012^{\mathrm{a}}$ \\
\hline MST, months & 9.00 & 12.00 & 20.50 & $<0.001^{\mathrm{a}}$ & $0.017^{\mathrm{a}}$ \\
\hline
\end{tabular}

${ }^{\mathrm{a}} \mathrm{P}<0.05$. TACE, trans-arterial chemoembolization; CIT, combined interventional therapy; OS, overall survival; MST, mean survival time.

implantation and 8 had RFA in addition to TACE for their liver metastases.

Tumor response to treatment. The tumor response to treatment was evaluated at 6 months after the intervention. The patients deceased at the time of the tumor response evaluation (i.e., at six months from the initiation of the treatment), in whom CT could not be performed to carry out tumor response evaluation, were not included in this analysis. The results are presented in Table II. In the control group 17.20\% of patients demonstrated CR or PR, 36.56\% had PD and 46.24\% had SD. In the TACE group, $30.61 \%$ of patients demonstrated CR or PR, 24.48\% had PD and 44.89\% exhibited SD. By contrast, $51.89 \%$ of patients experienced CR or PR in the CIT group. Furthermore, while only $20.25 \%$ had PD, $29.11 \%$ exhibited $\mathrm{SD}$ in the CIT group. The CR, PR, PD, and SD values of the CIT group were significantly different compared with control group ( $\mathrm{P}<0.05$ for each comparison). Furthermore, the overall response rate in the CIT group was revealed to be significantly higher compared with the control group $(\mathrm{P}<0.001)$. Although the individual comparisons of $\mathrm{CR}, \mathrm{PR}, \mathrm{PD}$ and $\mathrm{SD}$ between CIT and TACE groups exhibited no significant differences, the overall response rate between the two groups was significantly different $(\mathrm{P}=0.028)$.

Overall survival. The results for overall survival are presented in Table III. The overall survival rates at 12, 24 and 36 months were $23.57,9.75$ and $4.06 \%$ for the control group; 47.46, 25.42 and $11.86 \%$ for the TACE group; and and $67.85,39.28$ and $22.62 \%$ for the CIT group, respectively, as presented in Fig. 2 and Table II. The overall survival rate at 36 months for the CIT group (22.62\%) was significantly higher compared with the control group $(4.06 \% ; \mathrm{P}=0.0001)$ and the TACE group $(11.86 \% ; \mathrm{P}=0.0116)$. The median survival rate was $9.0,12.0$ and $20.5 \%$ for the control, TACE and CIT groups, respectively.

The present study further analyzed the differences in the overall survival rates for different combinations of interventional procedures for primary tumors. The difference in their overall survival rates at 36 months was insignificant (Fig. 3; TACE + RFA vs. TACE $\left.+{ }^{125} \mathrm{I} ; \mathrm{P}=0.382\right)$. However, the median survival rate for TACE $+{ }^{125} \mathrm{I}$ was 23.0 months, whereas that of TACE + RFA was only 15.5 months. As only 9 patients underwent TACE $+{ }^{125} \mathrm{I}+\mathrm{RFA}$, statistical analysis was not performed for this subgroup.

Subgroup analysis was also performed to evaluate the overall survival rate in patients with UR-L and UR-M. The median survival rates for all the treatment groups were significantly higher for UR-L patients compared with UR-M patients, which indicated that all treatments exhibited improved results for locally advanced tumor compared with metastatic tumor (Fig. 4).

Among UR-L patients, the median survival times were 11, 21 and 26 months for patients treated with systemic chemotherapy, TACE and CIT respectively. Among UR-M patients, the median survival times were 8.0, 11.0 and 19.5 months for patients treated with systemic chemotherapy, TACE and CIT, respectively. TACE and CIT exhibited significantly improved overall survival rates compared with systemic chemotherapy for treating both UR-M and UR-L patients (Fig. 4). Notably, 


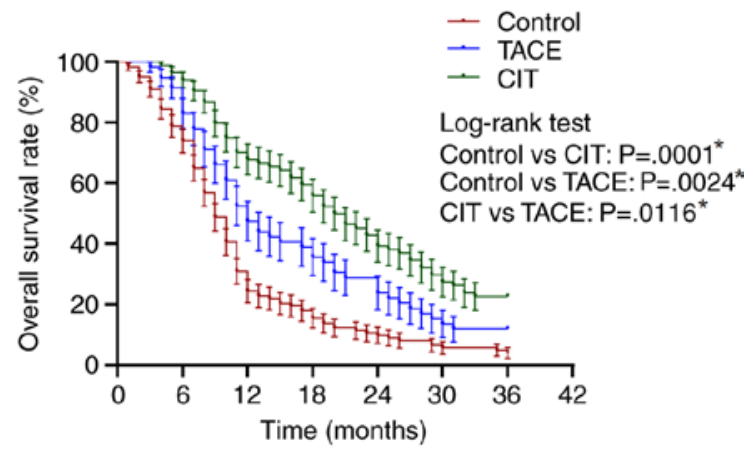

Figure 2. Kaplan-Meier curve demonstrating the 3-year overall survival rates of patients. A log-rank test indicated that the overall survival rate of the CIT group was significantly higher compared with the TACE group and the control group. ${ }^{*} \mathrm{P}<0.05$. CIT, combined interventional therapy; TACE, trans-arterial chemoembolization.

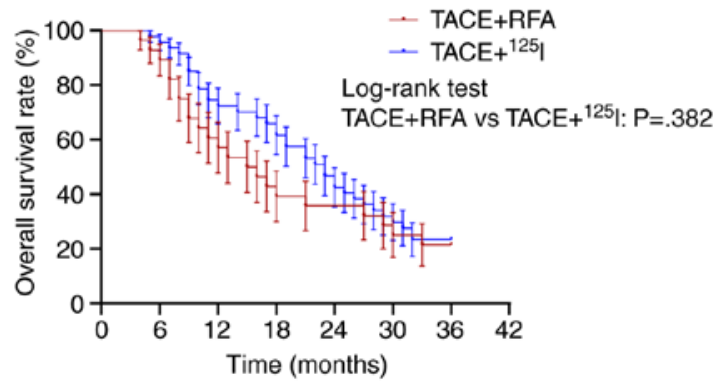

Figure 3. Kaplan-Meier curve demonstrating the 3-year overall survival rates of patients treated with different combinations of interventional therapy. A log-rank test indicated no significant difference in the overall survival rate between the TACE $+{ }^{125}$ I group and TACE + RFA group. TACE, trans-arterial chemoembolization; ${ }^{125}$ I, iodine-125; RFA, radiofrequency ablation.

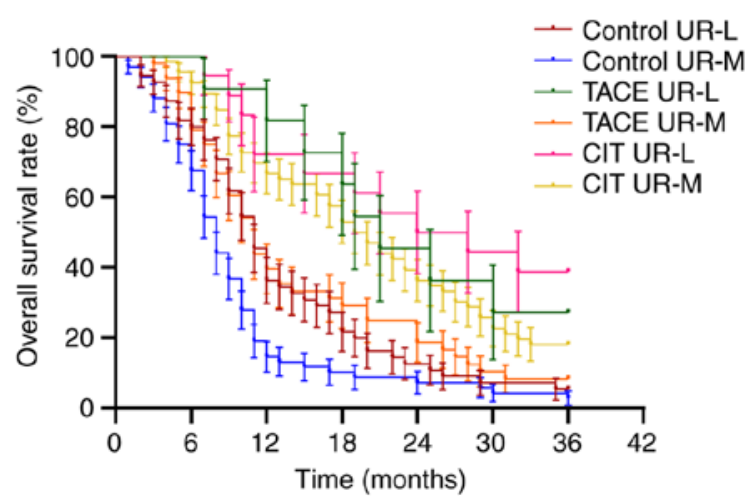

Log-rank test

Control UR-L vs Control UR-M: $P=.0128$ *

TACE UR-L vs TACE UR-M: $P=.0383^{*}$

CIT UR-L vS CIT UR-M: $P=.1041$

Control UR-L vs TACE UR-L: $P=.0093^{*}$

Control UR-L vs CIT UR-L: $P=.0004^{*}$

Control UR-M vs TACE UR-M: $P=.0052^{*}$

Control UR-M vs CIT UR-M: $P<.0001^{*}$

CIT UR-L vs TACE UR-L: $P=.6336$

CIT UR-M vs TACE UR-M: $P=.0088^{*}$

Figure 4. Kaplan-Meier curve demonstrating the 3-year overall survival rates of patients with UR-L and UR-M. A log-rank test indicated that the TACE and CIT groups had significantly higher overall survival rates compared with systemic chemotherapy for treating both UR-M and UR-L patients. "P<0.05. UR-L, locally advanced tumor; UR-M, unresectable metastatic tumor; TACE, trans-arterial chemoembolization; CIT, combined interventional therapy.

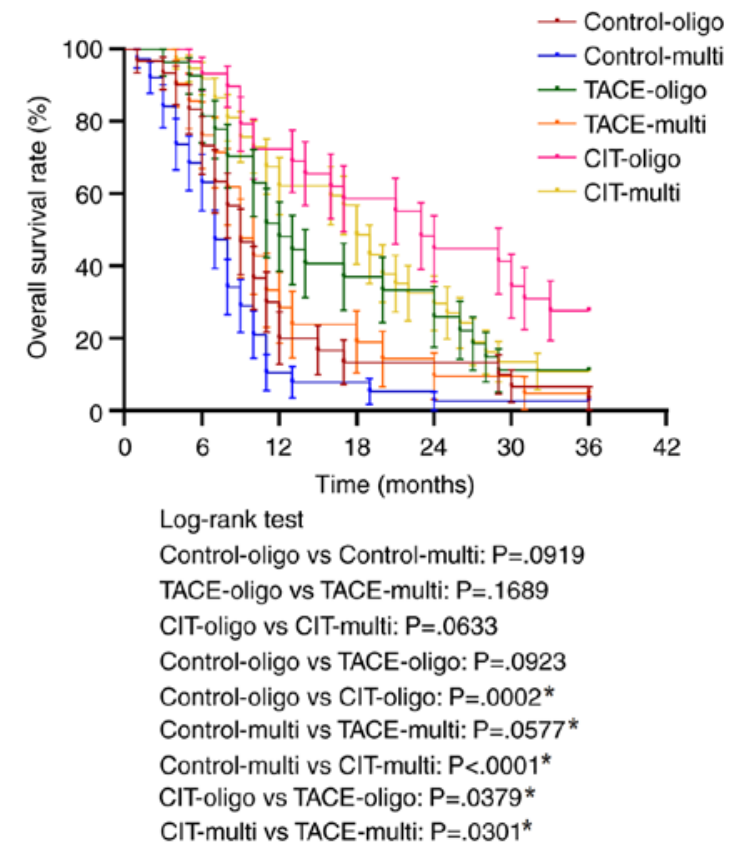

Figure 5. Kaplan-Meier curve demonstrating the 3 -year overall survival rates of patients with oligo ( $\leq 3$ liver lesions) or multi ( $>3$ liver lesions) metastases. A log-rank test indicated that the CIT group exhibited significantly higher survival rates for the treatment of oligo and multi metastases compared with either the control or TACE groups. "P $<0.05$. oligo, oligonodular; multi, multinodular; TACE, trans-arterial chemoembolization; CIT, combined interventional therapy.

CIT demonstrated a significantly improved overall survival rate for UR-M patients compared with TACE $(\mathrm{P}=0.0088)$.

In addition, the UR-M patients were analyzed to evaluate if the number of liver metastases affects the overall survival rate in each treatment group. The number of liver metastases (oligo nodular versus multi nodular metastases) did not affect the overall survival rate in either group. However, the CIT group exhibited significantly improved survival rates for treating both oligo nodular and multi nodular metastases compared with the control group and TACE group (Fig. 5).

Pain relief. Among all patients, 209 (78.57\%) were experiencing pain prior to treatment, of which $166(79.42 \%)$ demonstrated a good to medium response, while $43(20.57 \%)$ demonstrated a mild or no response. Among the control group, 61 patients $(65.59 \%)$ achieved a good or medium response, while 32 (34.41\%) exhibited a mild or no response. In the TACE group, 41 patients $(91.66 \%)$ achieved a good or medium response, and $7(14.58 \%)$ achieved a mild or no response following treatment. In the CIT group, 64 patients (94.12\%) achieved a good or medium response, and 4 patients (5.88\%) achieved a mild or no response following treatment. In summary, pain relief was more pronounced in the CIT group compared with the TACE group or control group.

Toxicity and complications. Few toxicity complications were observed in the present study and no patients died during the perioperative period in any of the groups. The drug and intervention-associated adverse effects reported with each treatment are presented in Table IV. In the control group, the most frequently reported non-hematological adverse events 
Table IV. Adverse events associated with the procedures.

\begin{tabular}{|c|c|c|c|c|}
\hline $\begin{array}{l}\text { Adverse } \\
\text { events }^{\mathrm{a}}\end{array}$ & $\begin{array}{c}\text { Systemic } \\
\text { chemotherapy }(\mathrm{n}=123), \mathrm{n}(\%)\end{array}$ & $\begin{array}{c}\text { TACE }^{\mathrm{b}} \\
(\mathrm{n}=143), \mathrm{n}(\%)\end{array}$ & $\begin{array}{c}{ }^{125} \mathrm{I} \text { seeds }{ }^{\mathrm{c}} \\
(\mathrm{n}=47), \mathrm{n}(\%)\end{array}$ & $\begin{array}{c}\mathrm{RFA}^{\mathrm{d}} \\
(\mathrm{n}=28, \mathrm{n}(\%)\end{array}$ \\
\hline \multicolumn{5}{|l|}{ Neutropenia } \\
\hline Grade 1 & $26(21.13)$ & $23(16.08)$ & $6(12.76)$ & $5(17.85)$ \\
\hline Moderate-grade 2 & $10(8.13)$ & $9(6.29)$ & $4(8.51)$ & $2(7.14)$ \\
\hline Grade 3 & $8(6.50)$ & $4(2.79)$ & $0(0.00)$ & $0(0.00)$ \\
\hline \multicolumn{5}{|l|}{ Anemia } \\
\hline Grade 1 & $18(14.63)$ & $21(14.68)$ & $7(14.89)$ & $6(21.42)$ \\
\hline Grade 2 & $7(5.69)$ & $11(7.69)$ & $5(10.63)$ & $0(0.00)$ \\
\hline \multicolumn{5}{|l|}{ Thrombocytopenia } \\
\hline Mild-grade 1 & $27(21.95)$ & $19(13.28)$ & $6(12.76)$ & $5(17.85)$ \\
\hline Moderate-grade 2 & $11(8.95)$ & 7 (4.89) & $2(4.25)$ & $1(3.57)$ \\
\hline \multicolumn{5}{|l|}{ Elevated AST } \\
\hline Mild-grade 1 & $25(20.32)$ & $29(20.27)$ & $5(10.63)$ & $5(17.85)$ \\
\hline Moderate-grade 2 & $9(7.31)$ & $14(9.79)$ & $3(6.38)$ & $2(7.14)$ \\
\hline Grade 3 & $0(0.00)$ & $3(2.09)$ & $1(2.12)$ & $1(3.57)$ \\
\hline \multicolumn{5}{|l|}{ Elevated ALT } \\
\hline Mild-grade 1 & $27(21.95)$ & $31(21.68)$ & $6(12.76)$ & $3(10.71)$ \\
\hline Moderate-grade 2 & $8(6.50)$ & $13(9.09)$ & $3(6.38)$ & $4(14.28)$ \\
\hline Grade 3 & $0(0.00)$ & $5(3.49)$ & $1(2.12)$ & $0(0.00)$ \\
\hline \multicolumn{5}{|c|}{ Increased serum bilirubin } \\
\hline Mild-grade 1 & $15(12.19)$ & $36(25.17)$ & $5(10.63)$ & $4(14.28)$ \\
\hline Grade 2 & $9(7.31)$ & $11(7.69)$ & $1(2.12)$ & $2(7.14)$ \\
\hline \multicolumn{5}{|l|}{ Pulmonary infection } \\
\hline Mild-grade 1 & $13(10.56)$ & $14(9.79)$ & $2(4.25)$ & $1(3.57)$ \\
\hline \multicolumn{5}{|l|}{ GI hemorrhage } \\
\hline Mild-grade 1 & $7(5.69)$ & $3(2.09)$ & $5(10.63)$ & $1(3.57)$ \\
\hline \multicolumn{5}{|c|}{ Pancreatic hemorrhage } \\
\hline Mild-grade 1 & $0(0.00)$ & $0(0.00)$ & $2(4.25 \%)$ & $0(0.00)$ \\
\hline \multicolumn{5}{|l|}{ Hepatic hemorrhage } \\
\hline Mild-grade 1 & $0(0.00)$ & $0(0.00)$ & $0(0.00)$ & $2(7.14)$ \\
\hline \multicolumn{5}{|l|}{ Fever } \\
\hline Grade 1 & $22(17.88)$ & $33(23.07)$ & $11(23.40)$ & $8(28.57)$ \\
\hline Grade 2 & $12(9.75)$ & $20(13.98)$ & $3(6.38)$ & $4(14.28)$ \\
\hline \multicolumn{5}{|l|}{ Fatigue } \\
\hline Grade 1 & $56(45.52)$ & $39(27.27)$ & $12(25.55)$ & $7(25.00)$ \\
\hline Grade 2 & $29(23.57)$ & $18(12.58)$ & $10(21.27)$ & $5(17.85)$ \\
\hline Grade 3 & $11(8.94)$ & $11(7.69)$ & $3(6.38)$ & $3(10.71)$ \\
\hline \multicolumn{5}{|l|}{ Nausea/vomiting } \\
\hline Grade 1 & $34(27.64)$ & $35(24.47)$ & $5(10.63)$ & $3(10.71)$ \\
\hline Grade 2 & $17(13.82)$ & $12(8.39)$ & $5(10.63)$ & $3(10.71)$ \\
\hline Grade 3 & $0(0.00)$ & $1(0.69)$ & $0(0.00)$ & $0(0.00)$ \\
\hline \multicolumn{5}{|l|}{ Pain } \\
\hline Grade 1 & $50(40.65)$ & $37(25.87)$ & $11(23.40)$ & $9(32.14)$ \\
\hline Grade 2 & $58(47.15)$ & $15(10.48)$ & $10(21.27)$ & $6(21.42)$ \\
\hline Grade 3 & $11(8.94)$ & $4(2.79)$ & $4(8.51)$ & $3(10.71)$ \\
\hline \multicolumn{5}{|l|}{ Peripheral neuropathy } \\
\hline Grade 1 & $35(28.45)$ & $6(4.19)$ & $2(4.25)$ & $1(3.57)$ \\
\hline Grade 2 & $13(10.56)$ & $0(0.00)$ & $0(0.00)$ & $0(0.00)$ \\
\hline Grade 3 & $6(4.87)$ & $0(0.00)$ & $0(0.00)$ & $0(0.00)$ \\
\hline
\end{tabular}

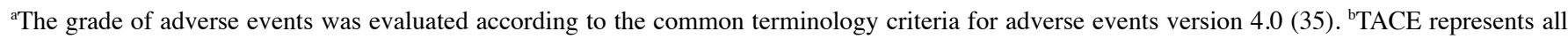
the patients undergoing TACE, either in the TACE alone group or combined interventional group. ${ }^{\mathrm{c} 25}$ I seeds represents all the patients undergoing ${ }^{125}$ I seed implantation, either alone or with TACE. ${ }^{\mathrm{R}} \mathrm{RFA}$ represents all the patients undergoing RFA, either alone or with TACE. TACE, trans-arterial chemoembolization; RFA, radiofrequency ablation; AST, aspartate transaminase; ALT, alanine transaminase. 
associated with treatment were fatigue (78.04\%), alopecia $(54.10 \%)$ and nausea (in $41.46 \%$ ). Reported treatment-associated adverse events of grade 3 or higher were neutropenia, fatigue and peripheral neuropathy. No patients in the control group discontinued treatment. A total of 14 patients (11.38\%) had at least one dose reduction of the chemotherapeutic agent in the control group.

Among the patients receiving TACE (either alone or in combination with other interventional treatment), the most frequently reported non-hematological adverse event associated with treatment was post-embolization syndrome, which is characterized by fever, nausea, vomiting, fatigue and elevation of liver enzymes. These patients were treated accordingly to manage their symptoms. Treatment-associated adverse events of grade 3 or higher were neutropenia, elevated liver enzymes and vomiting. Hospitalization with nasogastric tube feeding performed for patients with grade 3 vomiting.

Among patients treated with RFA (either alone or in combination with other interventional treatment), the most frequent non-hematological adverse events were associated with post-ablation/embolization syndrome, including pain, fever, nausea, vomiting and elevated liver enzymes. A total of 5 patients undergoing RFA developed hypertensive crisis, which includes a blood pressure $>220 / 115 \mathrm{mmHg}$ and a heart rate $>117$ beats $/ \mathrm{min}$. In each case, ablation was immediately discontinued, a selective $\alpha-1$ blocker was administered, blood pressure and heart rate normalized after $20 \mathrm{~min}$, and ablation was then continued. In total, 1 patient experienced intra-abdominal bleeding and 2 patients experienced hepatic bleeding, which improved upon management during the same hospitalization period.

Among patients treated with ${ }^{125}$ I seeds (either alone or in combination with other interventional treatment) implantation, pain, fever, nausea, vomiting and elevated liver enzymes were the most frequently reported adverse events. Reported treatment-associated adverse events of grade 3 or higher were neutropenia, pain, fatigue and elevated liver enzymes. During ${ }^{125}$ I seed implantation, transgression of the bowel during puncture did not result in substantial complications in the present study. However, a safer approach was achieved by traversing the stomach and avoiding the intestine and colon, particularly when using large-bore needles. A total of 17 patients exhibited a punctured visceral organ, including the liver (5), stomach (9) and intestine (3), during the implantation. Of these 17 patients, 0 had major adverse events; however, 5 of them experiences minor events. The minor events were slight gastrointestinal bleeding $(<20 \mathrm{ml})$ during implantation. In addition, 2 patients experienced intra-pancreatic bleeding, which was managed during the same hospitalization period. Furthermore, 2 patients had seeds that migrated to nearby organs; however, no side effects were observed in the 18 months post-treatment. Pain was the most common adverse effect in all the groups.

\section{Discussion}

The 5-year survival rate for patients with locally advanced pancreatic cancer is $\sim 4 \%$ and the overall survival time is 9-15 months, which may be reduced by 3-6 months in cases with metastases (37). Pancreatic cancer is as a major therapeutic challenge, which is largely characterized as a chemotherapy-refractory disease that exhibits a poor response to currently available treatments. The median overall survival time has been reported to be only 6.0-7.1 months for patients with advanced pancreatic cancer undergoing chemotherapy (38). Multimodal treatment protocols, including TACE, RFA and ${ }^{125}$ I radioactive seed implantation, have been established for the treatment of metastatic tumors. A continuous low-dose irradiation with ${ }^{125}$ I seeds causes Panc-1 cell-cycle arrest in the G2/M phase and induces apoptosis (39). Furthermore, implantation of ${ }^{125}$ I seeds as irradiation promotes the permeability of the surrounding vasculature and increases the efficacy of chemotherapy (40). In addition, thermal energy provided by RFA can cause cell death by coagulative necrosis and protein denaturation (41). It is also thought that RFA may have an immunogenic effect on tumors via an upregulation of heat shock protein 70 , which causes activation of dendritic cells, attracting infiltrating immune cells and eliciting a T cell response $(42,43)$. A systematic review of five studies that included 158 patients treated with RFA reported a positive impact on survival (44). Furthermore, image-guided radiotherapy is associated with an overall and progression-free survival time of 12.1 months and 7.6 months, respectively, for patients with pancreatic cancer (45). In summary, brachytherapy with ${ }^{125} \mathrm{I}$ seed implantation and RFA provides an option for patients with advanced pancreatic cancer who may benefit from combinational therapies. Therefore, the present study compared the efficacy of TACE with TACE + RFA and/or ${ }^{125}$ I radioactive seed implantation for unresectable pancreatic cancer. It was identified that TACE combined with either ${ }^{125}$ I seed implantation and/or RFA, improved the tumor treatment response and overall survival rate compared with TACE alone. This result suggested that RFA and radioactive seed implantation may be used to improve loco-regional disease control and improve survival compared with either systemic or intra-arterial chemotherapy alone for patients with pancreatic cancer.

In the present study, the CIT group demonstrated a significantly higher overall response rate compared with the TACE group (51.89 vs. $30.61 ; \mathrm{P}=0.028)$. The overall survival rate of CIT group was also higher compared with the TACE alone group at 3 years. This suggested that a combination of different interventional techniques may be effective for increasing the survival of patients with pancreatic cancer. Furthermore, all treatment groups demonstrated a higher overall survival rate for patients with locally advanced tumors compared with patients with metastatic tumors. Notably, CIT yielded a significantly improved overall survival rate for UR-M patients compared with TACE alone. However, CIT did not demonstrate any significant difference in the overall survival rate for treating oligo nodular or multi nodular metastatic patients. Overall, the present study revealed that combination therapy may be an effective option for the treatment of patients with unresectable pancreatic cancer. However, which specific combination achieves the best result could not be determined in the current study. The overall survival rate obtained with TACE $+{ }^{125}$ I was similar to that obtained with TACE + RFA. We hypothesize that the choice of TACE + RFA or TACE $+{ }^{125}$ I should depend on the condition of the patient, the location of the tumor and potential complications associated with the interventions. For example, tumors in close proximity with adjacent organs are at higher risk if treated 
with radioactive seeds. The present study could not conclude which combination is associated with more complications. Both the TACE $+{ }^{125} \mathrm{I}$ and TACE + RFA groups experienced various complications, which were managed accordingly in the present study. No life-threatening peri-procedural complications were associated with either of the groups. In the current study, only 9 patients received TACE $+{ }^{125} \mathrm{I}+\mathrm{RFA}$; therefore, the efficacy or the safety of this combination of treatment could not be analyzed due to the small number of patients.

There were some limitations of the present study. The retrospective nature and limited sample size may have reduced the generalizability of the results. Furthermore, there may have been a selection bias caused by non-randomization. In addition, the treatment protocol and the nature of health care delivery differs between different cancer institutions; therefore, the results based on a single center study may not be generalized to other institutions.

In conclusion, combined TACE $+{ }^{125} \mathrm{I}$ and/or RFA treatment may prolong the survival time compared with TACE alone for patients with unresectable pancreatic carcinoma. Notably, the use of concomitant RFA and ${ }^{125}$ I seed implantation may increase post-operative complications and the rate of adverse effects. However, the complications and adverse effects were demonstrated to be resolved following active management of the symptoms. Therefore, CIT may be a more optimal approach compared with a single interventional technique to achieve an improved tumor response and survival.

\section{Acknowledgements}

The authors wish to thank Professor Morgan A. McClure (Affiliated Hospital of North Sichuan Medical College, Nanchong, China) for his assistance with English grammar correction and revision.

\section{Funding}

No funding was received.

\section{Availability of data and materials}

The datasets used and/or analyzed during the current study are available from the corresponding author on reasonable request.

\section{Authors' contributions}

SKD, JLW and HFY conceived and designed the study. SKD, JLW, BL, CZ and HFY analyzed and interpreted the data. SKD, JLW, BL and CZ collected the data. BL and CZ performed statistical analysis. SKD wrote the manuscript. SKD, JLW and HFY critically revised the manuscript. All authors read and approved the final version of the manuscript.

\section{Ethics approval and consent to participate}

The current study was approved by the Institutional Review Board of Affiliated Hospital of North Sichuan Medical College (Nanchong, China) and Pingdingshan Fifth People's Hospital (Pingdingshan, China), and written informed consent was obtained from all patients.

\section{Patient consent for publication}

Not applicable.

\section{Competing interests}

The authors declare that they have no competing interests.

\section{References}

1. Siegel R, Ma J, Zou Z and Jemal A: Cancer statistics, 2014. CA Cancer J Clin 64: 9-29, 2014.

2. Zhang L, Jin H, Guo X, Yang Z, Zhao L, Tang S, Mo P, Wu K, Nie Y Pan Y and Fan D: Distinguishing pancreatic cancer from chronic pancreatitis and healthy individuals by (1) $\mathrm{H}$ nuclear magnetic resonance-based metabonomic profiles. Clin Biochem 45: 1064-1069, 2012.

3. Li D, Xie K, Wolff R and Abbruzzese JL: Pancreatic cancer. Lancet 363: 1049-1057, 2004.

4. Siegel R, Naishadham D and Jemal A: Cancer statistics, 2012. CA Cancer J Clin 62: 10-29, 2012.

5. Mao C, Domenico DR, Kim K, Hanson DJ and Howar JM: Observations on the developmental patterns and the consequences of pancreatic exocrine adenocarcinoma: Findings of 154 autopsies. Arch Surg 130: 125-134, 1995.

6. Kamisawa $\mathrm{T}$, Isawa $\mathrm{T}$, Koike $\mathrm{M}$, Tsuruta $\mathrm{K}$ and Okamoto A: Hematogenous metastases of pancreatic ductal carcinoma. Pancreas 11: 345-349, 1995.

7. Iacobuzio-Donahue CA, Fu B, Yachida S, Luo M, Abe H, Henderson CM, Vilardell F, Wang Z, Keller JW, Banerjee P, et al: DPC4 gene status of the primary carcinoma correlates with patterns of failure in patients with pancreatic cancer. J Clin Oncol 27: 1806-1813, 2009.

8. Frigerio I, Girelli R, Giardino A, Regi P, Salvia R and Bassi C: Short term chemotherapy followed by radiofrequency ablation in stage III pancreatic cancer: Results from a single center. J Hepatobiliary Pancreat Sci 20: 574-577, 2013.

9. Girelli R, Frigerio I, Salvia R, Barbi E, Tinazzi Martini P and Bassi C: Feasibility and safety of radiofrequency ablation for locally advanced pancreatic cancer. Br J Surg 97: 220-225, 2010.

10. Keane MG, Bramis K, Pereira SP and Fusai GK: Systematic review of novel ablative methods in locally advanced pancreatic cancer. World J Gastroenterol 20: 2267-2278, 2014.

11. Spiliotis JD, Datsis AC, Michalopoulos NV, Kekelos SP, Vaxevanidou A, Rogdakis AG and Christopoulou AN: Radiofrequency ablation combined with palliative surgery may prolong survival of patients with advanced cancer of the pancreas. Langenbecks Arch Surg 392: 55-60, 2007.

12. Matsui Y, Nakagawa A, Kamiyama Y, Yamamoto K, Kubo N and Nakase Y: Selective thermocoagulation of unresectable pancreatic cancers by using radiofrequency capacitive heating. Pancreas 20: 14-20, 2000.

13. Date RS and Siriwardena AK: Radiofrequency ablation of the pancreas. II: Intra-operative ablation of non-resectable pancreatic cancer. A description of technique and initial outcome. JOP 6: 588-592, 2005.

14. Landman J: Differences in immunologic response to cryoablation versus radiofrequency ablation in the treatment of renal cell carcinoma. ClinicalTrial, RCT No. NCT03409224, 2018.

15. Rombouts SJ, Vogel JA, van Santvoort HC, van Lienden KP, van Hillegersberg R, Busch OR, Besselink MG and Molenaar IQ: Systematic review of innovative ablative therapies for the treatment of locally advanced pancreatic cancer. Br J Surg 102: 182-193, 2015.

16. Wu PH, Pan CC, Huang ZL, Li W, Zhao M and Zhou ZW: Percutaneous radiofrequency ablation approach through the spleen: Initial case report for pancreatic tail gastrinoma. Chin J Cancer 29: 836-841, 2010.

17. Paiella S, Salvia R, Girelli R, Frigerio I, Giardino A, D'Onofrio M, De Marchi G and Bassi C: Role of local ablative techniques (radiofrequency ablation and irreversible electroporation) in the treatment of pancreatic cancer. Updates Surg 68: 307-311, 2016.

18. Yu YP, Yu Q, Guo JM Jiang HT, Di XY and Zhu Y: Effectiveness and security of CT-guided percutaneous implantation of 125I seeds in pancreatic carcinoma. Br J Radiol 87: 20130642, 2014. 
19. Vogl TJ, Mohamed SA, Albrecht MH, Gruber-Roh T, Lin $\mathrm{H}$ Nour Eldin NEA, Bednarova I, Naguib NN and Panahi B: Transarterial chemoembolization in pancreatic adenocarcinoma with liver metastases: MR-based tumor response evaluation, apparent diffusion coefficient (ADC) patterns, and survival rates. Pancreatology 18: 94-99, 2018.

20. Ceha HM, van Tienhoven G, Gouma DJ, Veenhof $\mathrm{CH}$ Schneider CJ, Rauws EA, Phoa SS and González González D: Feasibility and efficacy of high dose conformal radiotherapy for patients with locally advanced pancreatic carcinoma. Cancer 89 . 2222-2229, 2000.

21. Du YQ, Li ZS and Jin ZD: Endoscope-assisted brachytherapy for pancreatic cancer: From tumor killing to pain relief and drainage. J Interv Gastroenterol 1: 23-27, 2011.

22. Paulsen SD, Nghiem HV, Negussie E, Higgins EJ, Caoili EM and Francis IR: Evaluation of imaging-guided core biopsy of pancreatic masses. AJR Am J Roentgenol 187: 769-772, 2006.

23. Smith EH: Complications of percutaneous abdominal fine-needle biopsy. Review. Radiology 178: 253-258, 1991

24. Vogl TJ, Zangos S, Heller M, Hammerstingl RM, Böcher E, Jacob U and Bauer RW: Transarterial chemoperfusion with gemcitabine and mitomycin c in pancreatic carcinoma: Results in locally recurrent tumors and advanced tumor stages. Rofo 179 : 1181-1188, 2007 (In German).

25. Huang ZM, Pan CC, Wu PH, Zhao M, Li W, Huang ZL and Yi RY: Efficacy of minimally invasive therapies on unresectable pancreatic cancer. Chin J Cancer 32: 334-341, 2013.

26. Lencioni R and Llovet JM: Modified RECIST (mRECIST) assessment for hepatocellular carcinoma. Semin Liver Dis 30 52-60, 2010.

27. Karnofsky DA and Burchenal JH: Evaluation of chemotherapeutic agents. In: The Clinical Evaluation of Chemotherapeutic Agents in Cancer. Macleod CM (ed). Columbia University Press, New York, NY, p205, 1949.

28. Liu X, Yang X, Zhou G, Chen $Y$, Li C and Wang $X$ : Gemcitabine-based regional intra-arterial infusion chemotherapy in patients with advanced pancreatic adenocarcinoma. Medicine (Baltimore) 95: e3098, 2016.

29. Long J, Luo GP, Xiao ZW, Liu ZQ, Guo M, Liu L, Liu C, Xu J, Gao YT, Zheng Y, et al: Cancer statistics: Current diagnosis and treatment of pancreatic cancer in Shanghai, China. Cancer Lett 346: 273-277, 2014.

30. Seldinger SI: Catheter replacement of the needle in percutaneous arteriography; A new technique. Acta Radiol 39: 368-376, 1953.

31. Wu ZX, Yang XZ, Cai JQ, Liao LM, Yang L, Lin YN and Tan JM: Digital subtraction angiography and computed tomography angiography of predominant artery feeding pancreatic body and tail. Diabetes Technol Ther 13: 537-541, 2011.

32. Donatini B: A systematic study of the vascularisation of the pancreas. Surg Radiol Anat 12: 173-180, 1990.

33. 'Pain Intensity Instruments'. National Institutes of Health Warren Grant Magnuson Clinical Center. July 2003. http://mvltca net/Presentations/mvltca.pdf. Accessed August 5, 2018.
34. Wang H, Wang J, Jiang Y, Li J, Tian S, Ran W, Xiu D and Gao Y: The investigation of $125 \mathrm{I}$ seed implantation as a salvage modality for unresectable pancreatic carcinoma. J Exp Clin Cancer Res 32: 106, 2013.

35. Common terminology criteria for adverse events (version 4.0). https://evs.nci.nih.gov/ftp1/CTCAE/CTCAE_4.03/Archive/ CTCAE_4.0_2009-05-29_QuickReference_8.5x11.pdf. Accessed August 19, 2018.

36. Morin E, Cheng S, Mete O, Serra S, Araujo PB, Temple S, Cleary S, Gallinger S, Greig PD, McGilvray I, et al: Hormone profiling, WHO 2010 grading, and AJCC/UICC staging in pancreatic neuroendocrine tumor behavior. Cancer Med 2: 701-711, 2013.

37. Vincent A, Herman J, Schulick R, Hruban RH and Goggins M: Pancreatic cancer. Lancet 378: 607-620, 2011.

38. Saif MW: New developments in the treatment of pancreatic cancer. highlights from the '44th ASCO annual meeting'. Chicago, IL, USA. May 30-June 3, 2008. JOP 9: 391-397, 2008.

39. Wang J, Wang J, Liao A, Zhuang $\mathrm{H}$ and Zhao Y: The direct biologic effects of radioactive $125 \mathrm{I}$ seeds on pancreatic cancer cells PANC-1, at continuous low-dose rates. Cancer Biother Radiopharm 24: 409-416, 2009.

40. Cron GO, Beghein N, Crokart N, Chavée E, Bernard S, Vynckier S, Scalliet $\mathrm{P}$ and Gallez B: Changes in the tumor microenvironment during low-dose-rate permanent seed implantation iodine-125 brachytherapy. Int J Radiat Oncol Biol Phys 63: 1245-1251, 2005.

41. Nikfarjam M, Muralidharan V and Christophi C: Mechanisms of focal heat destruction of liver tumors. J Surg. Res 127: 208-223, 2005.

42. Teng LS, Jin KT, Han N and Cao J: Radiofrequency ablation, heat shock protein 70 and potential anti-tumor immunity in hepatic and pancreatic cancers: A minireview. Hepatobiliary Pancreat Dis Int 9: 361-365, 2010

43. Dromi SA, Walsh MP, Herby S, Traughber B, Xie J, Sharma KV, Sekhar KP, Luk A, Liewehr DJ, Dreher MR, et al: Radiofrequency ablation induces antigen-presenting cell infiltration and amplification of weak tumor-induced immunity. Radiology 251: 58-66, 2009.

44. Fegrachi S, Besselink MG, van Santvoort HC, van Hillegersberg R and Molenaar IQ: Radiofrequency ablation for unresectable locally advanced pancreatic cancer: A systematic review. HPB (Oxford) 16: 119-123, 2014.

45. Son SH, Song JH, Choi BO, Kang YN, Lee MA, Kang KM and Jang HS: The technical feasibility of an image-guided intensity-modulated radiotherapy (IG-IMRT) to perform a hypofractionated schedule in terms of toxicity and local control for patients with locally advanced or recurrent pancreatic cancer. Radiat Oncol 7: 203, 2012. 